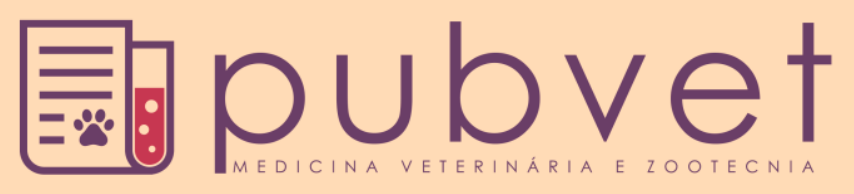

HTTP://DX.DOI.ORG/10.22256/PUBVET.V11N1.87-90

\title{
Ganho em peso médio diário de diferentes grupos genéticos de bovinos de corte
}

\author{
Aluísio de Alencastro Filho', Wilian Vaz Silva², Breno de Faria Vasconcelos ${ }^{3}$, Rodrigo \\ Zaiden Taveira $^{4 *}$, Felipe Eguti de Carvalho ${ }^{5}$
}

${ }^{I}$ Mestrando em ciências ambientais e Saúde da Pontifícia Universidade Católica de Goiás, PUC-GO Brasil. E-mail: aluisiofilho@zootecnista.com.br ${ }^{2}$ Docente adjunto do curso de Biologia. Escola de Ciências Agrárias e Biológicas (ECAB) - Pontifícia Universidade Católica de Goiás, PUC-GO Brasil. E-mail: herpetovaz@gmail.com

${ }^{3}$ Docente adjunto do curso de Zootecnia. Escola de Ciências Agrárias e Biológicas (ECAB) - Pontifícia Universidade Católica de Goiás, PUC - GO Brasil. Email:breno.ucg@gmail.com

${ }^{4}$ Docente e Pesquisador da Universidade Estadual de Goiás, Curso de Zootecnia. Campus de São Luis de Montes Belos - GO Brasil. E-mail. rodrigozaiden@gmail.com

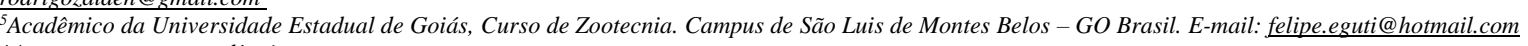
*Autor para correspondência

RESUMO. O ganho em peso médio diário consiste em importante ferramenta no monitoramento do desempenho de bovinos em confinamento, tendo em vista o aproveitamento dos alimentos oferecidos. Neste sentido, objetivou-se avaliar o ganho em peso médio de diferentes grupos genéticos de bovinos de corte submetidos à engorda em sistema de confinamento. Foram utilizadas informações de 17.704 bovinos machos não castrados de três grupos genéticos: Nelore, Aberdeen Angus e Composto Montana. Todos os animais foram alimentados com a mesma dieta e mantidos em confinamento por 124 dias até o abate. Os dados foram analisados pelo programa Statistical Analysis System (SAS) e as médias comparadas entre si por meio da utilização do teste de Tukey, ao nível de significância de $5 \%$. O ganho em peso médio diário foi de $1,50 \mathrm{~kg} / \mathrm{dia}, 1,33 \mathrm{~kg} / \mathrm{dia}$ e $1,43 \mathrm{~kg} / \mathrm{dia}$, para os grupos genéticos Aberdeen Angus, Nelore e Composto Montana respectivamente. $\mathrm{O}$ ganho médio diário foi maior para os animais do grupo genético Aberdeen Angus em comparação aos grupos genéticos Nelore e Montana.

Palavras chave: Confinamento, desempenho, produção animal.

\section{Evaluation of daily average weight gain from different genetics groups of beef cattle}

ABSTRACT. The daily average weight gain consists in important tool in the monitory of the performance of bovines kept in feedlot, considering the use of the aliments offered. In this way, the aim was to evaluate the average weight gain of different genetics groups of beef cattle submitted to finish beef in feedlot system. It was used information's provided by 17.704 bulls of three different genetic groups: Nellore, Aberdeen Angus e Montana breed. All of the animals were fed with the same diet and kept in feedlot for 124 days until the slaughter. The data was analysed by the Statistical Analysis System program and the means was compared by the use of Tukey test $(\mathrm{p}<0.05)$. The daily average weight gain was $1.50 \mathrm{~kg} / \mathrm{day}, 1.33 \mathrm{~kg} / \mathrm{day}$ and $1.43 \mathrm{~kg} /$ day, for the genetic groups Aberdeen Angus, Nellore and Compound Montana respectively. Average daily gain was higher to Aberdeen Angus genetic group in comparison with Nellore and Montana genetic groups.

Keywords: Feedlot, animal performance, animal production

Ganancia diaria de peso de tres grupos genéticos de bovinos de carne 
RESUMEN. La ganancia diaria de peso consiste en una importante herramienta en el monitoreo del desempeño de bovinos estabulados, teniendo en vista el aprovechamiento de los alimentos ofrecidos. En este sentido, el objetivo fue evaluar la ganancia media diaria de peso de diferentes grupos genéticos de bovinos de carne sometidos al engorde en sistema de establo. Fueron utilizadas informaciones de 17.704 bovinos machos no castrados de tres grupos genéticos: Nelore, Aberdeen Angus y Compuesto Montana. Todos los animales fueron alimentados con la misma dieta y mantenidos en corral de engorde por 124 días hasta el sacrificio. Los datos fueron analizados por el programa Statistical Analysis System (SAS) y los promedios comparados entre sí por el teste de Tukey, a nivel de significancia de $5 \%$. La ganancia diaria promedio de peso fue de $1,50 \mathrm{~kg} /$ día, 1,33 kg/día e 1,43 kg/día, para os grupos genéticos Aberdeen Angus, Nelore y Compuesto Montana respectivamente. La ganancia promedio de peso fue mayor para los animales del grupo genético Aberdeen Angus en comparación a los grupos genéticos Nelore y Montana.

Palabras clave: Cebadero, desempeño, producción animal.

\section{Introdução}

O confinamento é capaz de proporcionar segurança ao sistema de produção quando se deseja atingir determinados índices produtivos, tais como o controle da dieta, redução do tempo de abate e o monitoramento do desempenho dos animais (Costa et al., 2002; Prado, 2010). Nesse sentido, o ganho em peso médio diário apresentase como um importante índice de desempeno a ser estimado e avaliado nos sistemas de produção de bovinos de corte em confinamento (Rotta et al., 2009b). Em conjunto com os dias de confinamento avaliam o custo benefício da produção, auxiliando na tomada de decisão por parte dos gestores ( et al., 2010).

Objetivou-se identificar qual grupo genético apresenta maior ganho em peso médio diário quando submetidos a engorda em confinamento.

\section{Material e Métodos}

Foram utilizadas informações de 17.704 bovinos machos não castrados de três distintos grupos genéticos: Nelore (14.388 animais), Aberdeen Angus (163 animais) e composto Montana (3.153 animais), com idade média de 12 meses. Foram analisados o ganho em peso médio diário (GPMD) e o ganho em peso médio total (GPMT). Todos os animais foram alimentados com a mesma dieta e mantidos em confinamento por 124 dias até o abate.

Para verificar o efeito do grupo genético sobre o GMD e GPT foi realizada uma análise de variância. Sob modelo linear generalizado (GL). Nos casos em que houve diferença significativa entre os grupos, foi aplicado o teste de Tukey, ao nível de significância de 5\% para comparação de médias. Os dados foram analisados pelo programa Statistical Analysis System (SAS, 2004).

\section{Resultados e Discussão}

A tabela 1 apresenta a análise de variância do ganho em peso médio diário (GMD) tendo em vista os grupos genéticos avaliados. Considerando a avaliação do GMD dos bovinos em confinamento, pode ser notado que o grupo Aberdeen Angus, Nelore e composto Montana obtiveram 1,50 kg/dia, 1,33 kg/dia e 1,43 kg/dia, respectivamente, durante os 124 dias do confinamento, tendo sido constatado diferença estatisticamente significativa $(\mathrm{P}<0,05)$.

A tabela 2 apresenta os valores médios e desvios padrões do ganho em peso médio diário dos grupos genéticos Aberdeen Angus, Nelore e composto Montana.

Tabela 1. Análise de variância da variável ganha em médio diário (GMD) considerando os grupos genéticos avaliados.

\begin{tabular}{lccc}
\hline Fonte & $\begin{array}{c}\text { Graus de } \\
\text { liberdade }\end{array}$ & $\begin{array}{c}\text { Quadrado } \\
\text { médio }\end{array}$ & Pr $>$ F \\
\hline Grupo genético & 2 & 14.716179 & $<0.0001$ \\
Total & 17.703 & & \\
\hline
\end{tabular}

A superioridade dos animais Aberdeen Angus pode ser justificada pelos trabalhos de seleção realizados e também pela elevada taxa de ganho em peso, na composição dessa raça (Rotta et al., 2009a). Restle et al. (2002) explicam que as raças europeias são superiores as raças zebuínas como resultado da maior pressão de seleção para ganho e peso sofrida pelas primeiras, que se manifesta, principalmente, em boas condições alimentares.

Perotto et al. (2000) e Perotto et al. (2002) relataram valores bem próximos em animais Aberdeen Angus (1,478 kg/dia), o que assemelha aos resultados encontrados nessa pesquisa. Brondani et al. (2004) avaliando o ganho de peso 
diários de bovinos Aberdeen Angus encontrou valores inferiores $(1,350 \mathrm{~kg} / \mathrm{dia})$ e Costa et al. (2002) avaliando o desempenho de novilhos de Red Angus confinados até os $370 \mathrm{~kg}$ de peso vivo encontraram valores inferiores $(1,27 \mathrm{~kg} / \mathrm{dia})$. Silva et al. (2014) e Valero et al. (2015) encontraram valores bem próximos em animais Nelores não castrados (1,20 e 1,40 kg/dia, respectivamente). Isso assemelha com os resultados observados nessa pesquisa onde o ganho em peso foi de 1,33 $\mathrm{kg} / \mathrm{dia}$. Valores superiores também foram encontrados de $1,50 \mathrm{~kg} / \mathrm{dia}$, durante 84 dias de confinamento (Valero et al., 2015). Zawadzki et al. (2011) encontraram valores inferiores para o grupo genético Nelore abaixo de 1,10 kg/dia.

Tabela 2. Médias e desvio padrão do ganho em peso médio diário dos grupos genéticos Aberdeen Angus, Nelore e composto Montana.

\begin{tabular}{ll}
\hline Grupo Genético & Média \pm DP \\
\hline Aberdeen Angus & $1,50 \pm 0,32 \mathrm{a}$ \\
Nelore & $1,33 \pm 0,29 \mathrm{~b}$ \\
Composto & $1,43 \pm 0,33 \mathrm{c}$ \\
\hline
\end{tabular}

Médias seguidas por letras diferentes são diferentes pelo teste de Tukey $(\mathrm{P}<0,05)$

A raça é um fator que pode influenciar no ganho em peso. Vieira (2015) trabalhando com grupos genéticos Bosmara, Brangus e Canchim obteve resultados de GD de 1,79 kg/dia, 1,87 $\mathrm{kg} / \mathrm{dia}, 1,53 \mathrm{~kg} / \mathrm{dia}$, respectivamente. Esses resultados são superiores ao achado nesse trabalho. Leme (2001) trabalhando com grupos genéticos $1 / 2$ South Devon $v s$. Nelore não castrado $(1,32 \mathrm{~kg} / \mathrm{dia})$ e com $1 / 2$ Hereford $v s$. Nelore não castrados $(1,21 \mathrm{~kg} / \mathrm{dia})$ encontrou valores inferiores ao encontrado nessa pesquisa.

Rotta et al. (2009b) descrevem que os bovinos cruzados comparados aos Nelores, quando abatidos em idades semelhantes, possuem maior vantagens em relação ao peso vivo de abate quando abatidos em idades semelhantes, devido ao maior PVI e maior GD do que os cruzados em ralação aos nelores durante o confinamento. Já Estrada (1996), comparando NE com os seus mestiços meio-sangue ( $1 / 2$ Nelore x Normando, $1 / 2$ Nelore $x$ Holandês e $1 / 2$ Nelore $x$ Angus), pesquisou que os bovinos mestiços mostraram-se sem mais eficientes na engorda em confinamento, uma vez que obtiveram maior ganho de peso. Cruz et al. (2004) analisando o desempenho de animais puros Canchim e Nelore e cruzados, $1 / 2$ Limosin $x$ Nelore, $1 / 2$ Canchim x Nelore, $1 / 2$ Blonde x Nelore e $1 / 2$ Piemontês x Nelore, observou haver diferença significativa $(\mathrm{P}<0,05)$ entre os grupos genéticos na avaliação das medias estimadas para ganho de peso diário, com ganhos $1,66 \mathrm{~kg}, 1,12 \mathrm{~kg}, 1,70 \mathrm{~kg}$; $1,47 \mathrm{~kg} ; 1,55 \mathrm{~kg}$ e $1,48 \mathrm{~kg} \pm 0,04 \mathrm{~kg}$, respectivamente.

É de extrema importância que o produtor avalie o ganho em peso dos animais, por meio do ganho em peso médio diário, esperando-se que os animais mais eficientes ganhem mais peso num menor espaço de tempo (Prado, 2010). Conforme Hersom et al. (2004) o ganho em peso diário apresenta estimativa de correlação negativa com o tempo de permanência dos bovinos em confinamento. Portanto, de acordo com estes mesmos autores, a elevação do ganho em peso irá proporcionar redução no número de dias do confinamento.

No presente estudo, pode ser observado que os animais do grupo genético Aberdeen Angus, Nelore e Composto Montana obtiveram ganho em peso médio total de $184 \pm 39,30 \mathrm{~kg}, 167 \pm 35,90 \mathrm{~kg}$ e $164,6 \pm 36,70 \mathrm{~kg}$, respectivamente. Em sistemas de produção em confinamento o desempenho animal de acordo com Albright et al. (1993) é evidenciado, principalmente, pela avaliação do ganho em peso, o qual constitui-se num dos principais fatores que afetam a eficiência do sistema de produção.

\section{Conclusões}

Os animais do grupo genético Aberdeen Angus apresentaram desempenho superior, medido pelo ganho em peso médio diário, em detrimento aos animais Nelore e Composto Montana quando submetidos ao confinamento.

\section{Referências Bibliográficas}

Albright, M. L., Langemeier, M. R. Mintert, J. R. \& Schroeder, T. C. 1993. Factors affecting cattle feeding profitability and cost of gain. BCH-8050, Kansas State Univ., Manhattan.

Brondani, I. L., Sampaio, A. A. M. Restle, J. Rosa, J. R. P. Santos, C. V. M. Santos, M. F., Garagorry, F. C. \& Heck, I.. 2004. Desempenho de bovinos jovens das raças aberdeen angus e hereford, confinados e alimentados com dois níveis de energia. Revista Brasileira de Zootecnia, 33, 2308-17.

Costa, E.C., Restle, J., Vaz, F.N., Alves Filho, D.C., Bernardes, R. \& Kuss, F. 2002. Características da carcaça de novilhos Red Angus superprecoces abatidos com diferentes pesos. Revista Brasileira Zootecnia, 31, 119-8. 
Cruz, G. M., Tullio, R. R., Esteves, S. N., Alencar, M. M. \& Cordeiro, C. A., 2004. Peso de abate de machos não-castrados para produção do bovino jovem. 2. Peso, idade e características da carcaça. Revista Brasileira de Zootecnia, 33: 646-657.

Estrada, L. H. C. 1996. Composição corporal e exigências de proteína, energia e macro elementos minerais ( $\mathrm{Ca}, \mathrm{P}, \mathrm{Mg}, \mathrm{Na}$ e $\mathrm{K}$ ), características de carcaça e desempenho do Nelore e mestiços em confinamento. Tese (Doutorado em Zootecnia). Universidade Federal de Viçosa, UFV. p.128.

Hersom, M. J., Horn, G. W., Krehbiel, C. R. \& Phillips, W. A. 2004. Effect of live weight gain of steers during winter grazing: I. Feedlot performance, carcass characteristics, and body composition of beef steers. Journal Animal Science, 82: 262-272.

Leme, A.C.F. 2001. Produção e qualidade de carcaças de bovinos terminados em confinamento. Tese (Doutorado em Zootecnia). FCAV, UNESP, Jaboticabal. f.95.

Perotto, D., Cubas, A. C., Moletta, J. L. \& Lesskiu, C. 2000. Heterosis upon weights in Canchim and Aberdeen Angus calves and in their reciprocal crosses. Pesquisa Agropecuaria Brasileira, 35: 2511-2520.

Perotto, D., Moletta, J. L. \& Lesskiu, C. 2002. Desempenho em confinamento de machos bovinos inteiros canchim, Aberdeen Angus e cruzados recíprocos. Ciência Rural 32: 669674.

Prado, I. N. 2010. Produção de bovinos de corte e qualidade da carne. Eduem, Maringá, Paraná, Brasil.

Restle, J., Pascoal, L. L., Faturi, C., Alves Filho, D. C., Brondani, I. L., Pacheco, P. S. \& Peixoto, L. A. O. 2002. Efeito do grupo genético e da heterose nas características quantitativas da carcaça de vacas de descarte terminadas em confinamento. Revista Brasileira de Zootecnia, 31: 350-362.

Rotta, P. P., Prado, I. N., Prado, R. M., Moletta, J. L., Silva, R. R. \& Perotto, D. 2009a. Carcass characteristics and chemical composition of the Longissimus muscle of Nellore, Caracu and Holstein-friesian bulls finished in a feedlot. Asian-Australas Journal Animal Science, 22: 598-604.

Rotta, P. P., Prado, R. M., Prado, I. N., Valero, M. V., Visentainer, J. V. \& Silva, R. R. 2009b. The effects of genetic groups, nutrition, finishing systems and gender of Brazilian cattle on carcass characteristics and beef composition and appearance: a review. Asian-Australas Journal Animal Science, 22: 1718-1734.

SAS. 2004. SAS/STAT User guide, Version 9.1.2. SAS Institute Inc, Cary, NC, USA.

Silva, L. G., Torrecilhas, J. A., Ornaghi, M. G., Eiras, C. E., Prado, R. M. \& Prado, I. N. 2014. Glycerin and essential oils in the diet of Nellore bulls finished in feedlot: animal performance and apparent digestibility. Acta Scientrarium Animal Science, 36: 177-184.

Silva, R. R., Prado, I. N., Carvalho, G. G. P., Silva, F. F., Almeida, V. V. S., Santana Júnior, H. A., Paixão, M. L. \& Abreu Filho, G. 2010. Níveis de suplementação na terminação de novilhos Nelore em pastagens: aspectos econômicos. Revista Brasileira de Zootecnia, 39: 20912097.

Valero, M. V., Zeoula, L. M., Moura, L. P. P., Júnior, J. B. G. C., Sestari, B. B., \& Prado. I. N. 2015. Propolis extract in the diet of crossbred ( $1 / 2$ Angus vs. $1 / 2$ Nellore) bulls finished in feedlot: animal performance, feed efficiency and carcass characteristics. Semina: Ciências Agrárias, 36: 1067-1078.

Vieira, L. D. C. 2015. Desempenho de bovinos cruzados e parâmetros qualitativos de músculos maturados, Universidade Estadual Paulista, Jaboticabal.

Zawadzki, F., Prado, I. N., Marques, J. A., Zeoula, L. M., Rotta, P. P., Sestari, B. B., Valero, M. V. \& Rivaroli. D. C. 2011. Sodium monensin or propolis extract in the diets of feedlot-finished bulls: effects on animal performance and carcass characteristics. Journal Animal Feed Science, 20: 16-25.

Article History:

Received 10 October 2016

Accepted 31 October 2016

Available on line 14 November 2016

License information: This is an open-access article distributed under the terms of the Creative Commons Attribution License 4.0, which permits unrestricted use, distribution, and reproduction in any medium, provided the original work is properly cited. 